Bio - grafía. Escritos sobre la Biología y su Enseñanza. ISSN 2027-1034

Edición Extraordinaria. p.p. 363 - 371

Memorias del IX Encuentro Nacional de Experiencias en Enseñanza de la Biología y la Educación Ambiental. IV Congreso Nacional de Investigación en Enseñanza de la Biología.

\title{
LA DESCRIPCIÓN DEL CRECIMIENTO DE LAS PLANTAS EN ESTUDIANTES DE BÁSICA PRIM ARIA
}

THE DESCRIPTION OF THE GROWTH OF PLANTS IN ELEMENT ARY STUDENTS

\author{
Adriana Lucia Mendoza Díaz \\ Liliana Berón Carrillo \\ Henry Giovany Cabrera Castillo
}

\section{RESUMEN:}

Esta comunicación presenta los resultados iniciales de la investigación titulada "Promover la descripción a través de la enseñanza del crecimiento de las plantas" que servirá para la obtención del título de Maestría en Educación Énfasis Educación Matemática y Ciencias Experimentales de la Universidad del Valle Sede Tuluá. Se enfoca específicamente en la pregunta ¿De qué manera describen los estudiantes el crecimiento de las plantas? Se optó por una investigación cualitativa de tipo descriptivo-interpretativo. Se aplicó un cuestionario a estudiantes de segundo y tercer grado de primaria, en una de las sedes de una institución educativa del municipio de Tuluá. Para el procesamiento del análisis se realizó un pre-análisis, la explotación del material y se describieron e interpretaron los resultados obtenidos. Concluimos que esos resultados deben ser una oportunidad para que en las clases de biología se establezcan estrategias para la adquisición de la descripción como una habilidad y se haga énfasis en todos los aspectos que se involucran en el crecimiento de las plantas.

PALABRA CLAVE: Descripción, crecimiento plantas, enseñanza biología, habilidades

\section{ABSTRAC:}

This communication presents the initial results of the research entitled "Promoting the description through the teaching of the growth of plants" that will serve to obtain a Master's Degree in Education Emphasis Experimental Sciences and Mathematics Education of the University of the Valley, in the Tuluá's branch. Focuses specifically on the question: In what way students describe plant growth? We chose a descriptive qualitative research. A questionnaire was applied to students of second and third grade, in one of the branches of an educational institution in the municipality of Tuluá. For the processing of

\footnotetext{
${ }^{1}$ I.E. Julio César Zuluaga

2 I.E. Julio César Zuluaga

${ }^{3}$ Universidad del Valle
} 
Bio - grafía. Escritos sobre la Biología y su Enseñanza. ISSN 2027-1034

Edición Extraordinaria. p.p. 363 - 371

Memorias del IX Encuentro Nacional de Experiencias en Enseñanza de la Biología y la

Educación Ambiental. IV Congreso Nacional de Investigación en Enseñanza de la Biología.

the analysis was carried out a pre-analysis, the exploitation of the material and were described and interpreted the results obtained. We concluded that these results should be an opportunity for implementing strategies in the biology classes for the acquisition of the description as a skill and lead an emphasis on all the aspects that are involved in the growth of the plants.

KEYWORDS: Description, growth plants, teaching biology, abilities

\section{INTRODUCCIÓN}

En las escuelas rurales de Colombia, el programa Escuela Nueva es considerado un modelo flexible que está basado en principios del aprendizaje activo, enseñanza multigrado, estrategia de enseñanza con guías, relación escuela y comunidad, trabajo individual y colectivo; a través de un proceso de aprendizaje activo y participativo que promueva en los estudiantes habilidades para aplicar el conocimiento, el aprender a pensar, y solidaridad, destrezas básicas en lenguaje, matemáticas, ciencias sociales y ciencias naturales (Bustos, 2011; Schiefelbein, 1993; Barbosa, Pedraza \& Quijano, 2010).

En lo que respecta a la enseñanza de las Ciencias Naturales y específicamente la biología, el principal objetivo es lograr que los estudiantes desarrollen pensamiento científico, crítico y reflexivo, para que cuenten con una visión sistémica de mundo natural dentro del contexto de desarrollo humano integral, equitativo y sostenible, que les proporcione una concepción de sí mismos y de sus relaciones con la sociedad y la naturaleza (MEN, 2010).

De acuerdo con Harlen (2010), la enseñanza de las ciencias naturales en nivel de básica primaria enfrenta al docente a múltiples desafíos relacionados con la ciencia. El estudiante es inducido a realizar pequeñas observaciones del medio al que tiene acceso, dando inicio a sencillas investigaciones, que lo conducen a la creación de pequeñas ideas, que, relacionadas con la ciencia, pueden ser aún bastante tenues, en este sentido, la enseñanza de la biología está estrechamente relacionada con la reproducción, la evolución, el desarrollo, la interacción y el crecimiento de los seres vivos. En lo que respecta a este trabajo, nos enfocaremos en la temática del crecimiento de las plantas ya que es un pilar fundamental que se trabaja durante toda la escuela primaria, además, permitirá el reconocimiento del ser humano como parte esencial del mundo en el que interactúa y que se relacione con su entorno natural desde su contexto (Brito, 2012; Gómez, Sanmartí, \& Pujol, 2007).

En el ámbito de la enseñanza de la biología, se deben fomentar actividades tanto teóricas como prácticas que permitan el desarrollo de habilidades como la observación, la comparación, la clasificación y la descripción. Esta última es considerada como una habilidad cognitivo lingüística que consiste en enumerar cualidades y propiedades de objetos, hechos o fenómenos observables o incluso de modelos abstractos (Jorba, 2000; 
Bio - grafía. Escritos sobre la Biología y su Enseñanza. ISSN 2027-1034

Edición Extraordinaria. p.p. 363 - 371

Memorias del IX Encuentro Nacional de Experiencias en Enseñanza de la Biología y la Educación Ambiental. IV Congreso Nacional de Investigación en Enseñanza de la Biología.

Sanmartí, 2007; Bermúdez, Orozco \& Trujillo, 2009; Romero \& Lozano, 2010; Murillo \& Martínez, 2014).

Destacamos que es fundamental antes de iniciar el proceso de enseñanza del crecimiento de las plantas, determinar la manera como los estudiantes la describen, ya que esto será un insumo clave para diseñar propuestas de enseñanza que favorezcan su desarrollo y que a corto plazo les ayudará para la construcción de pensamiento crítico, científico y reflexivo; de acuerdo con lo anterior, la pregunta que orienta este trabajo es ¿De qué manera describen los estudiantes el crecimiento de las plantas?

\section{METODOLOGÍA}

Este trabajo estuvo orientado por un enfoque cualitativo de tipo descriptivo-interpretativo, (Sampieri, Fernández \& Batista, 2006) el cual permitió obtener las ideas de los estudiantes y hacer una descripción detallada, de acuerdo con las categorías preestablecidas que permitieron hacer comparaciones entre sí, fundamentando las respuestas plasmadas en los instrumentos empleados. Participaron del proceso 28 estudiantes de una de las sedes de una institución educativa del municipio de Tuluá (Valle del Cauca/Colombia), de los grados segundo y tercero, que cumplían con los siguientes criterios: edad (entre los 8 y 10 años), lectura y escritura, grado de escolaridad, y caligrafía legible.

El instrumento fue un cuestionario diseñado a partir de los criterios de la descripción propuestos por Jorba (2000) y López (1998), es decir, pertinencia, apreciación de cualidades y uso del vocabulario y las cinco primeras etapas del crecimiento de las plantas planteadas por Schoonhoven (1987), es decir, germinación, emergencia, primeras hojas, prefloración y floración. Estuvo conformado por tres situaciones problema y cuatro preguntas abiertas. En la aplicación del instrumento los estudiantes respondieron de manera individual y el promedio del tiempo empleado fue de 34 minutos. 
Bio - grafía. Escritos sobre la Biología y su Enseñanza. ISSN 2027-1034

Edición Extraordinaria. p.p. 363 - 371

Memorias del IX Encuentro Nacional de Experiencias en Enseñanza de la Biología y la

Educación Ambiental. IV Congreso Nacional de Investigación en Enseñanza de la Biología.

Tabla 1. Categorías preestablecidas sobre descripción y crecimiento de plantas

\begin{tabular}{|c|c|c|}
\hline CATEGORIA & SUBCATEGORIAS & CODIGOS \\
\hline \multirow{5}{*}{ 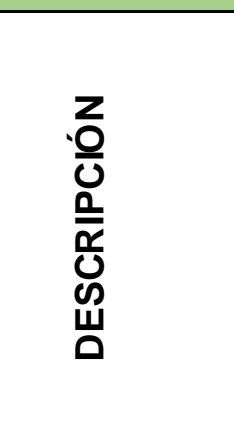 } & \multirow{2}{*}{ Pertinencia } & Precisión de la oración \\
\hline & & Imprecisión de la oración \\
\hline & Apreciación de cualidades & Calificativos \\
\hline & \multirow{2}{*}{ Uso de vocabulario } & Términos científicos \\
\hline & & Términos cotidianos \\
\hline \multirow{11}{*}{ 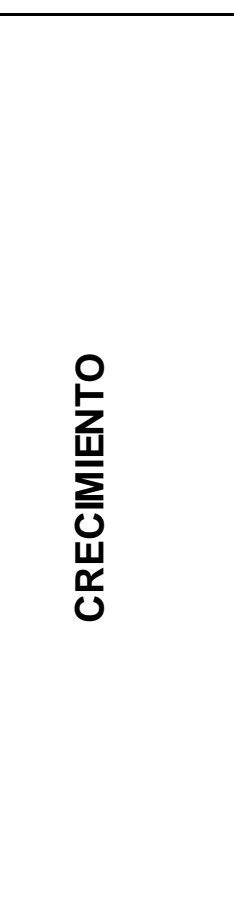 } & \multirow{3}{*}{ La Germinación } & Emerge la radícula \\
\hline & & Aparición raíz \\
\hline & & Crecimiento del hipocótilo a nivel de suelo \\
\hline & \multirow{3}{*}{ Emergencia } & Salida de la raíz \\
\hline & & Crecimiento del meristemo apical \\
\hline & & Aparición del tallo \\
\hline & \multirow{3}{*}{ Primeras hojas: } & Surgimiento de cotiledones \\
\hline & & Crecimiento del epicótilo \\
\hline & & Inicio de follaje \\
\hline & Prefloración & Aparición de botones de flor \\
\hline & Floración & Botones formados \\
\hline
\end{tabular}

Para el procesamiento de análisis se utilizó el software Atlas. Ti versión 7.5.4 con el fin de segmentar datos en unidades de significado, codificar datos y construir teoría, (Sampieri, Fernández \& Batista, 2006). Se utilizó la propuesta de Cabrera (2016) y constó de tres etapas: La primera etapa consistió en realizar un pre-análisis (lectura "superficial", elección de los documentos, constitución del corpus de documentos y preparación del material) para acercarse al material, determinar calidad y respuestas completas dadas por 
Bio - grafía. Escritos sobre la Biología y su Enseñanza. ISSN 2027-1034

Edición Extraordinaria. p.p. 363 - 371

Memorias del IX Encuentro Nacional de Experiencias en Enseñanza de la Biología y la

Educación Ambiental. IV Congreso Nacional de Investigación en Enseñanza de la Biología.

los estudiantes. La segunda etapa se enfocó en la explotación del material y consistió en desfragmentar en unidades de análisis, codificar y categorizar datos y describir las categorías predeterminadas (tabla 1). La tercera etapa se centró en describir e interpretar los resultados obtenidos a partir de la selección de unidades de análisis significativas.

\section{RESULTADOS Y DISCUSIÓN}

Debido a que los resultados del proceso de investigación y analítico son amplios, la discusión hará énfasis en únicamente en las subcategorías pertinencia y germinación. En cuanto a la subcategoría pertinencia, en las respuestas de los estudiantes se evidencia precisión ya que existe coherencia entre la escritura y la imagen o la situación planteada. En las siguientes unidades de análisis (tienen errores de ortografía porque se transcribieron como los estudiantes las escribieron), hemos señalado con morado el sujeto y con rojo el predicado:

"en esta imagen ya ba creciendo la rais del frijol"

"en la imagen 2 la semilla esta saliendo larraiz"

"la Planta crecio mucho"

Cabe resaltar que en los cuestionarios de algunos estudiantes se hizo más evidente los criterios de la subcategoría pertinencia, esto reafirma lo planteado por Jorba (2000) cuando dice que, el estudiante empleando sus conocimientos a través de la experiencia responde a una situación planteada donde evidencia el tema y el propósito del mismo, de igual manera, Espinal (2002), expresa que la oración es la unidad integrada por sujeto y predicado empleados para comunicar algo con entendimiento que permite enlazar el contenido con la forma, buscando las diferentes manifestaciones en una argumentación técnica y científica.

Dentro de las unidades de análisis codificadas como Imprecisión de la Oración, permite identificar los siguientes criterios como: No coincide la escritura con la imagen, no coincide la escritura con la situación planteada, no posee la estructura básica de una oración y/o tiene varios criterios de los antes mencionados. De acuerdo con la imagen 1 se seleccionan las unidades:
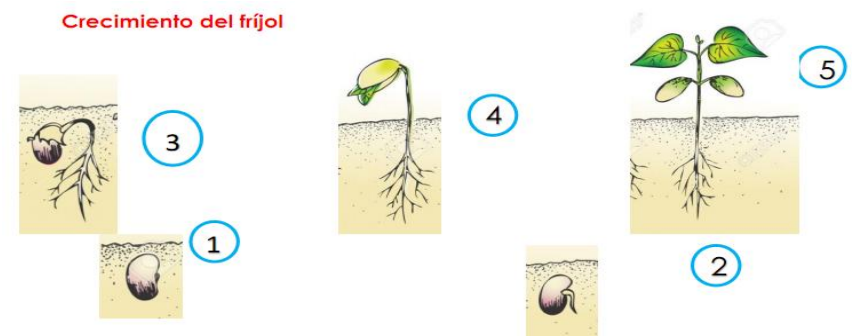

Imagen 1. Crecimiento del frijol 
Bio - grafía. Escritos sobre la Biología y su Enseñanza. ISSN 2027-1034

Edición Extraordinaria. p.p. 363 - 371

Memorias del IX Encuentro Nacional de Experiencias en Enseñanza de la Biología y la

Educación Ambiental. IV Congreso Nacional de Investigación en Enseñanza de la Biología.

No coincide la escritura con la imagen:

Imagen 1: "apenas bana sienóo la semillita".

Imagen 2: "esta creciendo el tallo"

Imagen 3: "esta en el crecimiento".

Imagen 4: "en esta otra imagen ya nomas ba a crecer".

Imagen 5: "xa nasieron los ojas x pipa lito x las raises".

No coincide la escritura con la situación planteada:

Imagen 1: "una cemilla".

No posee la estructura básica de una oración:

Imagen 1: "Una semilla".

Imagen 2: "una rra i s".

Imagen 3: "unca pu llo".

Imagen 4: "o tro ga pu llo".

Imagen 5: u ma ta de fri gol, una rra i s".

Posee varios criterios de los antes mencionados:

Imagen 1: "el brijol uno espera para que plante por que tomundo espera mide un anchito".

Imagen 2: "cvunlo lla el frijol crece".

Imagen 3: "y luego la raíz".

Imagen 4: "Y ademas sus frutos son muy hermosos son sus frutos Rojos".

Imagen 5: "el frijol ya no es un planta gran grande y cultive sus hojas".

Los estudiantes al describir el proceso inicial del crecimiento de una planta de frijol no evidencian pertinencia puesto que no utilizan lo términos dentro de una estructura de la oración, de lo cual permite inferir la necesidad de contar con imágenes que contengan amplitud de detalles y el contacto de los procesos de manera vivencial que le permitan al estudiante completar y ser más amplio en la información que escriba (López,1990), como 


\title{
Bio - grafía. Escritos sobre la Biología y su Enseñanza. ISSN 2027-1034
}

\section{Edición Extraordinaria. p.p. 363 - 371}

\author{
Memorias del IX Encuentro Nacional de Experiencias en Enseñanza de la Biología y la \\ Educación Ambiental. IV Congreso Nacional de Investigación en Enseñanza de la \\ Biología.
}

sucedió con la imagen 5 , la cual hace referencia al proceso final del crecimiento del frijol dentro del cuestionario planteado y en la cual los estudiantes fueron más acertados al construir unidades de análisis referidas al código de precisión de términos.

Por su parte, se identificó que en la sub categoría germinación, los estudiantes, se referían solamente a la aparición de la raíz de la planta, utilizando oraciones como "creceron las raizes a la planta" y "está saliendo la raíz", haciéndose repetitivas citas en las que se menciona el crecimiento, la salida y el nacimiento de la raíz, sin mencionar partes de la misma como su radícula e hipocótilo, de acuerdo con Barrera, López y Morales y García (2013), se puede evidenciar, que en la enseñanza de la germinación en básica primaria, se ha trabajado la germinación de forma superficial, haciéndose evidente la carencia de enseñanza de las diferentes etapas por las que pasan las semillas para lograr este proceso; así como, los factores internos y externos de las semillas, por lo que es de esperarse que el estudiante no incluyera estos elementos en sus respuestas.

En cuanto a los códigos emergencia de la radícula y el crecimiento del hipocótilo a nivel de suelo, en las respuestas dadas por los estudiantes no se identificaron Unidades de Análisis. Teniendo en cuenta a Gonzáles (2013), la enseñanza de las ciencias implica que se debe llevar al estudiante a fundar modelos significativos relevantes que lo lleven a dar explicación del entorno que lo rodea, es necesario utilizar estrategias en el aula que lo dirijan hacia la construcción de estos conocimientos.

\section{CONCLUSIONES}

Los resultados obtenidos y su correspondiente análisis y discusión permiten establecer una serie de conclusiones cada una de ellas relacionadas con la pregunta que orientó esta comunicación, es decir ¿De qué manera describen los estudiantes el crecimiento de las plantas? De acuerdo con esto se puede establecer que:

En relación con la subcategoría pertinencia se puede concluir que en las respuestas dadas por los estudiantes la construcción de oraciones se hace con sentido completo y claridad cuando se presentan imágenes con amplitud de detalles, igualmente, la experiencia y contacto directo con las plantas la toman como referencia para elaborar las oraciones. Es fundamental desarrollar la "descripción" como una habilidad cognitivo lingüística en los niños entre los 8 a 10 años ya que le permitirá desarrollar su pensamiento científico, holístico, reflexivo, critico dentro de su contexto; proporcionando cohesión y coherencia en las producciones textuales y orales, accediendo a la argumentación de manera pertinente en cualquier ciencia.

En cuanto a la germinación se sustenta que las respuestas dadas por los estudiantes se asocian con la imagen que observan, más no con conceptos propios de la disciplina, describiéndola como un proceso que concluye con el surgir de la raíz, además, es importante que en el proceso de enseñanza se incluyan e integren las fases de emergencia de la radícula y el crecimiento del hipocótilo a nivel de suelo, ya que son fundamentales para ampliar el conocimiento. 
Bio - grafía. Escritos sobre la Biología y su Enseñanza. ISSN 2027-1034

Edición Extraordinaria. p.p. 363 - 371

Memorias del IX Encuentro Nacional de Experiencias en Enseñanza de la Biología y la Educación Ambiental. IV Congreso Nacional de Investigación en Enseñanza de la Biología.

Finalmente, estos resultados obtenidos deben significar una oportunidad para que en las clases de biología se establezcan estrategias para la adquisición de la descripción como una habilidad y se haga énfasis en todos los aspectos que se involucran en el crecimiento de las plantas. De igual manera, los profesores de biología también deben ser formados para que puedan formular estrategias didácticas, desarrollen materiales que permitan la adquisición y desarrollo tanto de esta habilidad como de otras que están asociadas a ellas.

Agradecimiento: Las dos primeras autoras de este trabajo agradecen al Ministerio de Educación Nacional colombiano por la beca para la excelencia otorgada para realizar esta investigación.

\section{REFERENCIAS BIBLIOGRÁFICAS}

Barbosa, E., Pedraza, L. \& Quijano, M. (2010). El conocimiento didáctico de un docente del modelo escuela nueva. Segundo congreso nacional de investigación en educación en ciencias y tecnología. Seminario internacional sobre enseñanza de las ciencias, junio, 1-11.

Barrera, M., López S., \& Morales, S. (2013). El proceso de germinación: modelos expresados por estudiantes de básica primaria. (Trabajo de grado). Universidad de Antioquia, Medellín.

Bermúdez, O., Orozco, J., \& Trujillo, D. (2009). Leer y escribir comprensivamente en la escuela III: La descripción y la narración en el aula (Tesis Doctoral) Universidad Tecnológica de Pereira, Colombia.

Brito, S. (2012). La construcción del modelo ser vivo por alumnos de primaria: una propuesta e análisis basada en una progresión de aprendizaje (Tesis Doctoral). Universidad Autónoma de Barcelona, España.

Bustos, J. (2011). Investigación y escuela rural: ¿irreconciliables? Profesorado. Revista de Curriculum y Formación de Profesorado, 15(2), 155-170.

Cabrera, H. (2016). Aportes a la enseñanza de la química a partir de un estudio histórico filosófico de la experimentación asociada a la combustión para profesores en formación inicial (Tesis Doctoral). Universidad del Valle, Cali.

Espinal, O. (2002). Algunas consideraciones sobre la gramática y sus implicaciones para la enseñanza aprendizaje de la producción escrita. Ciencia y sociedad, 24(7), 578590.

Gómez, A., Sanmartí, N., \& Pujol, R. (2007) Fundamentación teórica y diseño de una unidad didáctica para la enseñanza del modelo ser vivo en la escuela primaria. Enseñanza de las ciencias, 25(3), 325-340.

González, G. (2013). El modelo de ser vivo: una secuencia indagativa con alumnado del grado de Educación Primaria. Enseñanza de las ciencias, (Extra), 2075-2081. 
Bio - grafía. Escritos sobre la Biología y su Enseñanza. ISSN 2027-1034

Edición Extraordinaria. p.p. 363 - 371

Memorias del IX Encuentro Nacional de Experiencias en Enseñanza de la Biología y la

Educación Ambiental. IV Congreso Nacional de Investigación en Enseñanza de la Biología.

Harlen, W. (2010). Principios y grandes ideas de la educación en ciencias. Gran Bretaña: Ashford Colur Press.

Jorba, J., Gómez, I., \& Prat, À. (Eds.). (2000). Hablar y escribir para aprender: uso de la lengua en situación de enseñanza-aprendizaje desde las áreas curriculares. Madrid: Síntesis.

López, L. (1990). Sabes enseñar a describir, definir, argumentar. La Habana: Pueblo y educación.

MEN. (2010). Manual de implementación Escuela Nueva. Bogotá: MEN

Murillo, J. A. y Martínez, C A. (2014). Habilidades de pensamiento social: Describir, explicar, interpretar y argumentar en el aula. Itinerario Educativo, (64), 108-125

Romero, E. \& Lozano, A. (2010). Adquisición de las habilidades lingüísticas y cognitivas, relevancia para el aprendizaje del lenguaje escrito. Umbral Cientifico, (16), 8-12.

Sampieri, R.., Collado, C., Lucio, P., \& Pérez, M. (2006). Metodología de la investigación. México: Mcgraw-hill.

Sanmartí, N. (2007). Hablar, leer y escribir para aprender ciencia. Publicado en: Fernández, P. (Ed). La competencia en comunicación lingüística en las áreas del currículo. Colección Aulas de Verano. Madrid: MEC.

Schiefelbein, E. (1993). En busca de la escuela del siglo XXI: ¿Puede darnos la pista la Escuela Nueva de Colombia? Santiago de Chile: UNESCO/UNICEF.

Schoonhoven, A. (1987). Sistema estándar para la evaluación de germoplasma de frijol. Cali: CIAT. 